\title{
硫-氟的邻位交叉效应
}

李懿伦, 余意, 袁耀锋 ${ }^{*}$, 叶克印 ${ }^{*}$

福州大学化学学院, 福州 350108

摘要: 乙烷分子中相邻的两个氢原子被氟取代以后, 它的最优构象就从全交叉式转变为邻位交叉, 这就是邻位交叉 效应影响分子构象最具代表性的一个例子。本文简要回顾了含氟邻位交叉效应的发展历史与重要应用, 重点介绍了 物理有机科研前沿的硫-氟邻位交叉效应。结合当前最新的科研成果, 验证了通过改变硫原子的电正性可以达到有效 调控邻位交叉效应这一重要策略。通过科研反哺教学, 有助于进一步加深对硫-氟邻位交叉效应的理解和认识。

关键词: 邻位交叉效应; 立体电子效应; 构象

中图分类号: G64; O6

\section{The Sulfur-Fluorine Gauche Effect}

\author{
Yilun Li, Yi Yu, Yaofeng Yuan *, Keyin Ye * \\ College of Chemistry, Fuzhou University, Fuzhou 350108, China.
}

\begin{abstract}
The most favored anti-conformation of ethane would turn into gauche-conformation when the vicinal hydrogen atoms are replaced by two fluorine atoms. Such a switch shows how the gauche effect impacts the relative conformation of organic molecules. Herein, we briefly survey the history of the gauche effect as well as its broad applications in organic synthesis. Particularly, we highlight the recent investigations on the related sulfur-fluorine gauche effect. With the aid of experiments, we confirmed that the sulfur-fluorine gauche effect could be easily regulated via the subtle change of positive charges on the sulfur atom. Such a positive interplay between scientific research and organic course teaching would profoundly help students to understand the basic concepts and advanced applications of the sulfur-fluorine gauche effect.
\end{abstract}

Key Words: Gauche effect; Stereoelectronic effect; Conformation

\section{1 前言}

立体化学是有机化学十分重要的一个组成部分 ${ }^{[1]}$ 。通过基础有机化学的学习, 我们知道乙烷分 子的优势构象是一个全交叉式构象(图1a)。其中, 乙烷分子两个碳原子上的氢原子距离最远, 分子处 于这种构象时最稳定。类似的, 正丁烷由于两个甲基取代基间的位阻相互排斥作用, 其最优构象是 两个甲基处于对交叉位置(图1b)。因此, 我们似乎可以得出这样一个结论一一分子中两个会形成较大 排斥的取代基总是倾向处于距离最远的位置，形成一个对位交叉的优势构象。

然而, 1,2 -二氟乙烷与乙烷分子的情况截然不同。1,2-二氟乙烷中, 两个可能会造成电荷排斥的 氟原子并不处于对位, 而是选择了一个邻交叉构象(图1 c)。1,2-二氟乙烷的这种邻交叉构象看似违反 常识, 但却可以很好地用立体电子效应进行解释。立体电子效应作为超共轭效应概念的延伸, 可以

收稿: 2021-10-28; 录用: 2021-11-29; 网络发表: 2021-12-24

“通讯作者, Emails: yaofeng_yuan@fzu.edu.cn (袁耀锋); kyye@fzu.edu.cn (叶克印)

基金资助：福州大学一流学科本科教学改革建设项目; 中国高等教育学会2021年度理科专项课题(21LKYB07) 
解释有机化学中很多的 “反常” 现象 ${ }^{[2]}$, 包括糖化学中的异头碳效应(anomeric effect)、米氏酸的反常 酸性 ${ }^{[3]}$ 等。近期的计算化学研究表明, 乙烷邻位交叉构象为优势构象也与 $\mathrm{C}-\mathrm{H}$ 键的超共轭效应有着 密切的关系 ${ }^{[4-6]}$ 。在 1,2 -二氟乙烷中, 氟原子的强电负性(3.98)使得 $\mathrm{C}-\mathrm{F}$ 键被强烈极化, 进而导致 $\mathrm{C}-\mathrm{F}$ 键 的反键轨道能量显著地下降, 最终使得 $\mathrm{C}-\mathrm{H}$ 键成键轨道对其的超共轭效应显著增强(图 $1 \mathrm{~d})$ 。在 $1,2-二$ 氟乙烷的邻位交叉优势构象中, 两根 $\mathrm{C}-\mathrm{F}$ 键都能受到其对位的 $\mathrm{C}-\mathrm{H}$ 键超共轭的强稳定化作用。这类 使分子邻位交叉构象(gauche conformation)优于对交叉构象(anti conformation)的作用称为邻位交叉效 应(gauche effect)。

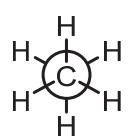

a. 对交叉

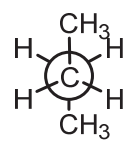

b. 对交叉

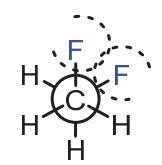

c. 邻交叉

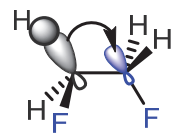

d. 立体电子效应模型

图1 取代基对烷烃优势构象的影响

实际上, 有关邻位交叉效应的研究早在 20 世纪中期就有所报道 ${ }^{[7]}$ 。1955年, Edward教授 ${ }^{[8]}$ 在吡喃 糖的研究中发现了异头碳效应。1,2-二氟乙烷的邻位交叉优势构象的发现, 则开启了含氟邻位交叉效 应研究发展的新篇章。在此之后, 更多的第二周期非金属元素与氟的邻位交叉效应(包括氟-氮、 氟-氧等)相继被研究报道。2015年, Gilmour教授课题组 ${ }^{[9]}$ 有关硫-氟邻位交叉效应的研究, 将杂原子 与氟的邻位交叉效应拓展到第三周期的硫元素, 进而促进了硫-氟邻位交叉效应的研究。本文基于 Gilmour教授课题组近期的相关研究, 对于硫-氟邻位交叉效应的研究进行简要的回顾和讨论。我们 希望通过基础理论的学习和最新科研成果的有机结合, 促进化学相关专业的本科生拓展知识面, 培养 他们理论联系实际、分析问题和解决问题的能力。

\section{2 硫-氟的邻位交叉效应}

\section{1 硫-氟邻位交叉效应的研究}

2015年, 德国明斯特大学的Gilmour教授课题组 ${ }^{[9]}$ 对氟硫醚化合物1 (图2)的晶体数据分析时 发现, FCCS的二面角为 $-62.07^{\circ}$, 接近邻位交叉构象的标准二面角 $\left(60^{\circ}\right)$ 。与此同时, 该分子中 $\mathrm{C}-\mathrm{H}$ 键 和 $\mathrm{C}-\mathrm{F}$ 键处于反式共平面 $\left(\phi_{\mathrm{HCCF}}=179^{\circ}\right)$, 存在明显的超共轭作用。晶体学数据进一步表明, 分子中 的两根 $\mathrm{C}-\mathrm{S}$ 键有着不同的键长, $\mathrm{C}_{4}-\mathrm{S}$ 键比 $\mathrm{C}_{1}-\mathrm{S}$ 键长 $2.7 \mathrm{pm}$, 这可能与 $\mathrm{C}_{5}-\mathrm{C}_{6}$ 键成键轨道对 $\mathrm{C}_{4}-\mathrm{S}$ 键 反键轨道的超共轭效应以及硫与 $\mathrm{C}_{4}$ 位上大位阻基团的排斥有关。同时, 通过 NMR对溶液中相应底物 的构象分析也表明, 相应分子在液相中也具有明显的邻位效应(化合物 $1 、 2$ 和 3 邻位交叉构象占比分 别为 $81.3 \%, 94.7 \%, 92.3 \%)$ 。

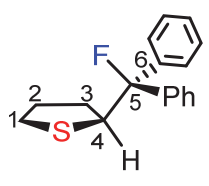

(1)

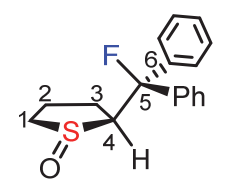

(2)

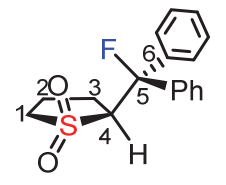

(3)

图 2 环状硫-氟化合物的邻位交叉效应

通常来说, 与碳原子相连的杂原子 $(\mathrm{X})$ 电负性越强时, 该 $\mathrm{C}-\mathrm{X}$ 键的极性越强, 从而使得 $\mathrm{C}-\mathrm{X}$ 键 反键轨道能量大幅度降低, 进而增强相邻成键轨道对其的超共轭作用。这一规律在硫-氟邻位交叉效 应中也得到了明显的体现。将硫醚底物选择性氧化成亚砜 $\left(\mathrm{S}=\mathrm{O}\right.$, 化合物 2 )和砜 $\left(\mathrm{SO}_{2}\right.$, 化合物 3$)$ 后, 相应的 $\mathrm{C}_{4}-\mathrm{S}$ 键与 $\mathrm{C}_{1}-\mathrm{S}$ 键的键长差值明显增大(键长差值分别为 $3.9 \mathrm{pm}, 5.4 \mathrm{pm}$ )。因此, 随着硫原子 
上的电正性增强, 硫-氟的邻位交叉效应更加明显。

进一步的DFT计算结果表明(图 3), 化合物 $\mathbf{4}$ 的对交叉构象比邻位交叉构象稳定(能量差为 $0.7 \mathrm{~kJ} \cdot \mathrm{mol}^{-1}$ )。将硫原子上的取代基由甲基换成对硝基苯基时(化合物 $\mathbf{5}$ ), 随着硫原子上的正电性增 加, 邻交叉构象开始成为优势构象 (能量差为 $0.1 \mathrm{~kJ} \cdot \mathrm{mol}^{-1}$ )。将化合物 4 中硫原子上的取代基替换为多 种具有不同电子效应的取代基，作者进一步考查了相应的线性自由能关系 ${ }^{[10]}$ 。对于硫醚和砜类底物, 邻位交叉构象占比基本与取代基吸电子能力呈正相关(回归系数分别为 $0.83 ， 0.80$ )。但对于亚砜类底 物, 邻位交叉构象占比与取代基吸电子能力不存在明显的正相关(回归系数仅为 0.26 ), 这可能与亚砜 自身较大的偶极对分子构象的影响有关。这些结果表明, 硫-氟的邻位交叉效应是包括超共轭效应、 偶极作用、静电作用等多种复杂因素相互作用共同导致的结果。

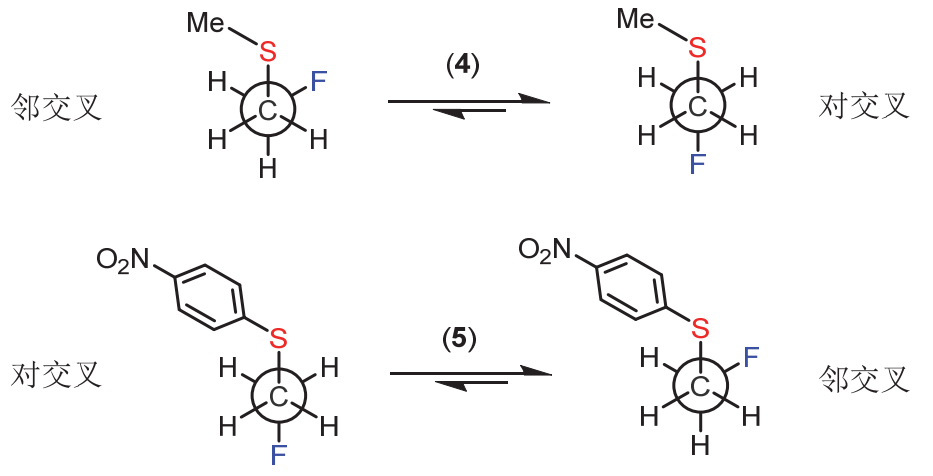

图3 取代基对硫-氟邻位交叉效应的影响

\section{2 通过过渡金属配位调控邻位交叉效应}

基于上述讨论可知, 提高硫原子的电正性 ${ }^{[11]}$, 可以显著增强硫-氟邻位交叉效应。与过渡金属配 位是提高硫原子电正性有效的方法之一。因此, 研究人员选取了环状硫醚底物, 考查其在与金、银 等金属配位后邻位交叉构象占比的变化。核磁数据表明(图4), 化合物6和化合物7在溶液中邻位交叉 构象的占比分别为 $90.3 \%$ 和 $98 \%$, 显著高于未与过渡金属配位前该硫化合物的邻位交叉构象占比 $(81.3 \%)$ 。这些结果表明, 通过配位方法提高硫原子的正电性的策略, 可以实现硫-氟邻位交叉效应的 选择性调控。基于以上研究, 我们猜想如果通过硫醚质子化的方式来提高硫原子的电正性(化合物8), 理论上也能够增强硫-氟邻位交叉效应。虽然该设想尚未得到实验的验证, 可以预见的是通过调节硫原 子的电正性增强硫-氟邻位交叉效应的策略将会得到进一步的发展, 在相关领域将会有更广泛的应用。

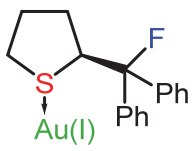

(6)

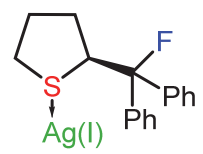

(7)

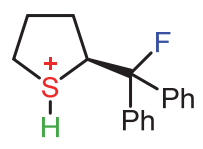

(8)

图4 硫的配位对硫-氟邻位交叉效应的影响

\section{3 含氟邻位交叉效应的实际应用}

氟的邻位交叉效应在有机催化、生物分子设计、材料化学等诸多领域有着十分广阔的应用前 景 ${ }^{[9]}$ 。例如, Gilmour教授课题组 ${ }^{[12,13]}$ 巧妙地利用了氟的邻位交叉效应对 J $\phi$ rgensen-Hayashi催化剂 进行了修饰。通过在手性胺催化剂中引入氟原子, 实现了肉桂醛类底物的高立体选择性环氧化反 应(图 5)。在催化剂的笁选过程中, 他们发现将氟原子替换为氢、苯基等取代基时, 产物的对映选择 性控制均大幅降低。作者推测, 氟的邻位交叉效应以及苯环与烯胺的 $\pi-\pi$ 堆积效应, 使催化剂中的苯 
环停留在烯胺上方从而阻碍了亲核试剂从烯胺上方的进攻, 进而大幅度提高了反应的立体选择性。

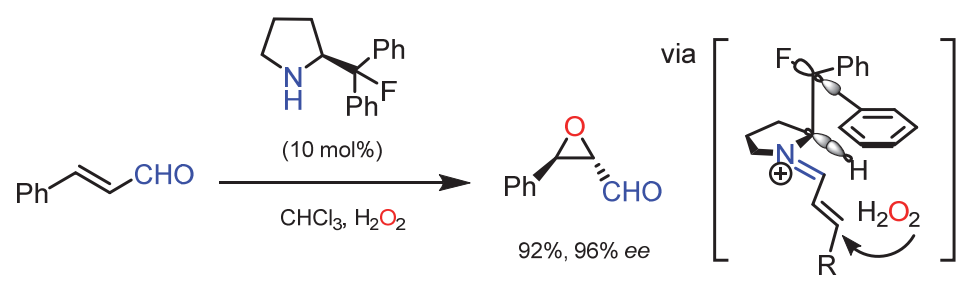

图5 邻位交叉效应在不对称催化中的应用

近期, 本课题组基于电化学合成手段实现了烯烃的高选择性氟-硫醚、氟-亚砜化反应 ${ }^{[14]}$, 该方 法为硫-氟邻位交叉效应的研究对象一一不同氧化态的邻位硫-氟类化合物提供了一种便捷的合成手 段。对相应产物的单晶数据分析表明, 与氟-硫醚产物(化合物9)相比, 氟-亚砜产物(化合物10)能够观 测到明显的硫-氟邻位交叉效应(图6)。这些实验结果与提高硫原子的电正性可以显著增强硫-氟邻位 效应的结论是一致的。

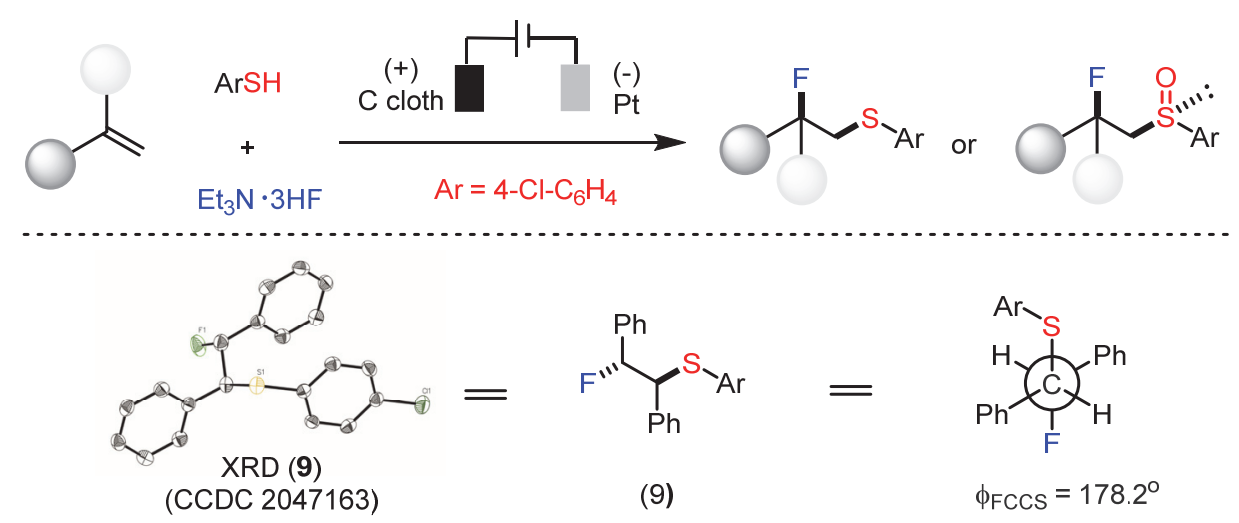

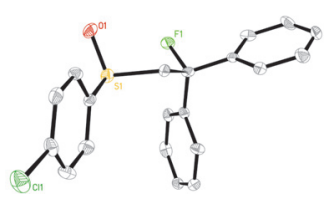

XRD (10) (CCDC 2045872)

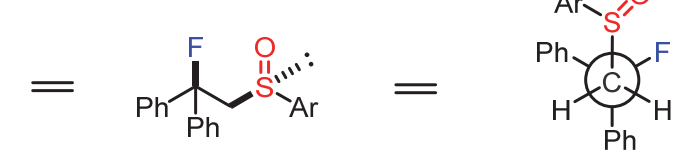

(10)

$$
\phi_{\mathrm{FCCS}}=50.6^{\circ}
$$

图6 电化学合成在硫-氟邻位交叉效应研究中的应用

\section{3 结语}

作为立体电子效应的重要组成部分, 硫-氟邻位交叉效应为许多有机化学中看似反常的立体化学 现象提供了良好的解释。随着理论化学与计算化学的进一步发展, 对硫-氟的邻位交叉效应的研究必 将得到进一步的拓展, 进而促进硫-氟邻位交叉效应在相关领域的广泛应用。

\section{参 考 文 献}

[1] 王辉, 曾卓. 大学化学, 2016, 31 (11), 22.

[2] 洪鹄, 黄兆和, 柴正琪, 张沅珒. 大学化学, 2017, 32 (1), 84. 
[3] 吕萍, 王彦广. 大学化学, 2018, 33 (12), 113.

[4] 周公度. 大学化学, 2001, $16(5), 51$.

[5] Pophristic, V.; Goodman, L. Nature 2001, 411, 565.

[6] Weinhold, F. Nature 2001, 411, 539.

[7] Thiehoff, C.; Rey, Y. P.; Gilmour, R. Isr. J. Chem. 2017, 57, 92.

[8] Edward, J. T. Chem. Ind. 1955, 1102.

[9] Thiehoff, C.; Holland, M. C.; Daniliuc, C.; Houk, K. N.; Gilmour, R. Chem. Sci. 2015, 6, 3565.

[10] Thiehoff, C.; Schifferer, L.; Daniliuc, C. G.; Santschi, N.; Gilmour, R. J. Fluorine Chem. 2016, 182, 121.

[11] Santschi, N.; Thiehoff, C.; Holland, M. C.; Daniliuc, C. G.; Houk, K. N.; Gilmour, R. Organometallics 2016, 35, 3040.

[12] Aufifiero, M.; Gilmour, R. Acc. Chem. Res. 2018, 51, 1701.

[13] Tanzer, E.-M.; Zimmer, L. E.; Schweizer, W. B.; Gilmour, R. Chem. - Eur. J. 2012, $18,11334$.

[14] Yu, Y.; Jiang, Y.-M.; Wu, S.-F.; Shi, Z.-J.; Wu, J.-N.; Yuan, Y.; Ye, K.-Y. Chin. Chem. Lett. 2022, 33 (4), 2009. 